\title{
Augmentation in restless legs syndrome: poor response to sudden withdrawal of dopaminergic therapy
}

This article was published in the following Dove Press journal: Journal of Parkinsonism and Restless Legs Syndrome 25 July 2013

Number of times this article has been viewed

\section{Roger Kurlan \\ Marcie Rabin}

Atlantic Neuroscience Institute, Overlook Medical Center, Summit, NJ, USA
Correspondence: Roger Kurlan Atlantic Neuroscience Institute, Overlook Medical Center, Summit, NJ 0790I, USA

Tel +l 9085222089

$\mathrm{Fax}+\mid 9085226147$

Email roger.kurlan@atlantichealth.org
Background: Augmentation is a common complication of long-term dopaminergic therapy for restless legs syndrome (RLS).

Methods: We conducted a retrospective chart review of patients with RLS and augmentation to learn more about the condition, including response to therapy.

Results: We identified 14 patients (nine women, five men; mean age 65.7 years; mean duration of RLS 23.6 years) with augmentation. Thirteen patients were taking a dopamine agonist and one was taking levodopa. Mean duration of dopaminergic therapy was 5.5 years. The dopaminergic drug was withdrawn at one time and replaced by gabapentin $(n=9)$, tramadol $(n=5)$, clonazepam $(n=4)$, propoxyphene $(n=2)$, or codeine $(n=1)$. Seven patients required more than one drug to control symptoms. The mean time until augmentation resolved was 12.5 (range 1-43) weeks, shortest when gabapentin was used first (2.6 weeks) and longer when clonazepam (14.9 weeks) or tramadol (22.3 weeks) was the initial replacement drug.

Conclusion: Augmentation was the most common problem associated with referral of a patient with RLS to a movement disorders specialist, yet referring physicians did not appear to know how to treat it. Regaining satisfactory control of symptoms following sudden withdrawal of the offending dopaminergic drug was difficult, taking longer than 3 months on average. The optimum treatment of drug-induced augmentation is unclear and needs further investigation.

Keywords: restless legs syndrome, dopamine agonists, levodopa, augmentation

\section{Introduction}

Restless legs syndrome (RLS) is characterized by uncomfortable feelings in the legs that typically develop soon after lying down at night for sleep and that lead to a sense of restlessness and an urge to move the legs, arise and walk about, or engage in other activities to relieve the restlessness. RLS is estimated to occur in moderate to severe levels of severity in $2.7 \%$ of the population. ${ }^{1}$ The primary disability of RLS is loss of sleep with daytime tiredness. Dopaminergic medications (usually dopamine agonists or levodopa) are the most predictably beneficial drugs for the treatment of RLS. However, their long-term use is associated with potential complications, particularly tolerance and augmentation. ${ }^{2}$ Tolerance refers to the tendency for the benefits to wear off over time, resulting in the need for gradually increasing doses. Eventually, with higher doses, medication side effects may outweigh any benefits. Augmentation is a phenomenon in which the medications cause a worsening of symptom severity above and beyond the level at the time the medication was started. Evidence of augmentation includes earlier onset of symptoms (in the evening before lying down for bed or in the daytime), a shorter time period between laying down for sleep and onset of RLS symptoms, 
increased severity of leg discomfort, restlessness or urge to move, involvement of previously unaffected limbs, such as the arms, and/or a shortened duration of treatment effect. Formal diagnostic criteria for augmentation in RLS have been published and include response to dopaminergic medication. ${ }^{3}$ We have recently encountered several patients experiencing dopaminergic drug-induced augmentation and found that this complication of therapy was difficult to treat.

\section{Materials and methods}

We conducted a retrospective chart review of all patients with a diagnosis of RLS seen at the Movement Disorders Program of the Atlantic Neuroscience Institute between 2010 and 2012. Information on patient demographics and history of RLS and its treatment were abstracted and summarized. We focused on the time period from the diagnosis of druginduced augmentation to its resolution.

\section{Results}

We identified 14 patients referred for RLS. All patients were referred by internists or general neurologists. In every case, the referring problem was worsening RLS and the primary problem turned out to be dopaminergic drug-induced augmentation. The diagnosis of augmentation was based on published diagnostic criteria. ${ }^{3}$ At the time of presentation, the mean International RLS Study Group Rating Scale score ${ }^{4}$ was 28.2 (range 24-36) and the Augmentation Severity Rating Scale score $^{5}$ was 9.2 (range 6-21). In reviewing office notes and letters of referral, none of the referring physicians mentioned the phenomenon of augmentation. There were five males and nine females with a mean age of 65.7 (range 36-88) years. The mean duration of RLS was 23.6 (range 1-60) years. At the time of presentation, seven patients were taking ropinirole at a mean dose of 1.7 (range 0.5-6) $\mathrm{mg}$ /day, six were taking pramipexole at a mean dose of 0.9 (range $0.125-2.5$ ) $\mathrm{mg} /$ day, one was taking both dopamine agonists, and one was being treated with levodopa (750 mg/day). One patient was taking tramadol and hydrocodone in addition to ropinirole. The mean duration of therapy with a dopaminergic drug was 5.5 (range 1.5-10) years.

In each case, at the time of the initial visit we discontinued the dopaminergic drug without tapering the dose and replaced it with one drug from a different class of medications (antiepileptics [gabapentin], benzodiazepines [clonazepam], narcotic analgesics [codeine, tramadol, propoxyphene]), used to treat RLS. The replacement drug choice was based on selecting a drug class that the patient was not currently being treated with, one that he or she was not recently treated with (to avoid the problem of tolerance) and one that the subject did not experience a poor response to or side effects in the past. Additional drugs were added over time as needed to control symptoms. Replacement medications included gabapentin (mean dose $525 \mathrm{mg}$ /day; range 100-1500 mg) in nine patients, tramadol (mean dose $180 \mathrm{mg} /$ day; range $100-250 \mathrm{mg}$ ) in five patients, clonazepam (mean dose $0.88 \mathrm{mg} /$ day; range $0.5-1.5 \mathrm{mg}$ ) in four patients, propoxyphene (no longer marketed; mean dose $97.5 \mathrm{mg} /$ day; range $65-130 \mathrm{mg}$ ) in two patients, and codeine ( $60 \mathrm{mg} /$ day) in one patient. Seven patients required one replacement medication, four required two medications, and three required three medications to achieve resolution of the augmentation.

Upon withdrawal of the dopaminergic drug, all but three of the patients experienced an initial worsening of RLS symptoms that was often quite severe, with major impairment in sleep, until augmentation resolved. Three of the patients were lost to follow-up after the initial evaluations so their outcomes are unknown. For the remaining 11 patients, the mean time period for resolution of augmentation after withdrawal of the offending drug was 12.5 (range 1-43) weeks. The mean time to resolution was 2.6 (range 1-6) weeks when gabapentin was the initial replacement drug $(n=4)$, 14.9 (range 1-43) weeks when clonazepam was used first $(n=3)$, and 22.3 (range 10-31) weeks when tramadol was the first drug $(n=3)$. One patient was started on propoxyphene and had resolution of augmentation after 14.3 weeks. The initial replacement drug was the only medication needed to control symptoms in three quarters of the patients started on gabapentin, two thirds of those started on tramadol, and one third of those started on clonazepam. The patient started on propoxyphene needed a second medication.

\section{Discussion}

While there are published studies assessing the prevalence of augmentation in larger samples of RLS patients, ours is the first study to investigate the time period following identification of augmentation systematically to determine the impacts of dopaminergic medication withdrawal and replacement drug therapy. Our study is limited by its retrospective approach and relatively small sample size. In addition, follow-up information was unavailable for three of the subjects. Despite these shortcomings, our study yielded several interesting and important observations. First, dopaminergic drug-induced augmentation was the most common (sole) reason for referring a patient with RLS to a movement disorders specialist. Second, since all referrals came from a practitioner of internal medicine or a general neurologist, 
it appears that nonspecialty physicians are comfortable diagnosing the condition and prescribing therapy. Third, our referring physicians unfortunately did not seem to know how to prevent or treat augmentation, despite the fact that it is a common complication of therapy. One 10-year longitudinal assessment of patients with RLS found that 5\%-7\% of those treated with a dopamine agonist developed augmentation each year, with only $35 \%-58 \%$ of these patients able to stay on these drugs for 10 years. ${ }^{6}$ It has been estimated that $76 \%$ of RLS patients treated with dopaminergic therapy develop augmentation and that dopamine agonists appear to have a limited period of utility for many patients. ${ }^{7}$

Our review of medical records indicated that referring physicians tended to increase dopaminergic drug doses steadily when faced with augmentation. This is in contradiction to the published expert recommendations to discontinue the offending medication and switch to a different drug or drug class. ${ }^{2,3,8}$ However, it should be noted that, in the published literature on RLS, there appears to be little discussion of the best approach to treating augmentation once it appears, including how best to withdraw the dopaminergic medication.

Our findings indicate that once augmentation develops, the process of switching to an alternative drug class is difficult. Most patients experienced a substantial worsening of RLS and sleep deprivation upon withdrawal of the offending dopaminergic drug. On average, it took more than 3 months to re-establish satisfactory control of symptoms, often requiring use of multiple medications. Only three of our subjects were successfully transitioned to another drug within a 2-week period. The underlying neurobiologic mechanisms of dopaminergic drug-induced augmentation remain unknown, although alterations in dopamine receptor number or sensitivity are likely to be involved and such changes could take extended periods of time to correct.

Our very preliminary findings suggest that control is achieved faster when gabapentin is used initially as the replacement drug, compared with clonazepam or tramadol. More research is needed, however, to confirm this observation. While keeping dopaminergic drug doses low and initiating drug class rotation upon observing tolerance or at the first sign of augmentation has been suggested as a strategy to minimize or avoid augmentation, ${ }^{2,3,9}$ the optimal approach for treating this problem once it develops remains to be determined. In our patients, sudden withdrawal of the dopaminergic drug was used. It is possible that this sudden withdrawal leads to a worsening of RLS similar to what has been described for the motor features of Parkinson's disease. ${ }^{10}$ Perhaps a gradual tapering of the drug dose until it is discontinued would be better tolerated. Alternatively, gaining control of symptoms with an alternative drug prior to tapering or stopping the dopamine agonist might be a better option. Clearly, this aspect of RLS therapy requires more study.

In summary, physicians should be aware that treating RLS with dopaminergic drugs is not simple and that careful long-term monitoring of medication responses is needed. Before prescribing, all physicians should be cognizant of the potential complications of tolerance and augmentation and how to deal with them. More research is needed to identify the optimal approach for treating augmentation once it develops. Sudden withdrawal of the offending dopaminergic drug does not appear to be a well tolerated strategy. The potentially difficult problem of augmentation during therapy with a dopaminergic medication should be carefully considered when selecting a medication for the treatment of RLS.

\section{Disclosure}

Both authors recently participated in a clinical trial of rasagiline for restless legs syndrome supported by Teva Neuroscience Inc. RK is a member of the speakers bureau for Teva Neuroscience Inc.

\section{References}

1. Allen R, Walters AS, Montplaisir J. Restless legs syndrome prevalence and impact. Arch Intern Med. 2005;165:1286-1292.

2. Kurlan R, Richard IH, Deeley C. Medication tolerance and augmentation in restless legs syndrome: the need for drug class rotation. $J$ Gen Intern Med. 2006;21:C1-C4.

3. Allen RP, Pichietti D, Hening WA, Trenkwalder C, Walters AS, Montplaisir J. Restless legs syndrome: diagnostic criteria, special considerations, and epidemiology. A report from the restless legs syndrome diagnosis and epidemiology workshop at the National Institutes of Health. Sleep Med. 2003;4:101-119.

4. Walters AS, LeBrocq C, Dhar A, et al. Validation of the International Restless Legs Syndrome Study Group rating scale for restless legs syndrome. Sleep Med. 2003;4:137-141.

5. Garcia-Borreguero D, Kohnen R, Hogl B, et al. Validation of the Augmentation Severity Rating Scale (ASRS): a multicentric, prospective study with levodopa on restless legs syndrome. Sleep Med. 2007;8:455-463.

6. Silver N, Allen RP, Senerth J, Earley CJ. A 10-year, longitudinal assessment of dopamine agonists and methadone in the treatment of restless legs syndrome. Sleep Med. 2011;12:440-444.

7. Garcia-Borreguero D, Hogl B, Ferini-Strambi L, et al. Systematic evaluation of augmentation during treatment with ropinirole in restless legs syndrome (Willis-Ekbom disease): results from a prospective, multicenter study over 66 weeks. Mov Disord. 2012;27:277-283.

8. Williams A-M, Garcia-Borreguero D. Management of restless legs syndrome augmentation. Curr Treat Options Neurol. 2009;11:327-332.

9. Oertel WH, Trenkwalder C, Zucconi M, et al. State of the art in restless legs syndrome therapy: Practice recommendations for treating restless legs syndrome. Mov Disord. 2007;22 Suppl 18:S466-S475.

10. Rabinak CA, Nirenberg MJ. Dopamine agonist withdrawal syndrome in Parkinson disease. Arch Neurol. 2010;67:58-63. 
Journal of Parkinsonism \& Restless Legs Syndrome

\section{Publish your work in this journal}

Journal of Parkinsonism and Restless Legs Syndrome is an online, open access, peer-reviewed journal. The journal publishes review articles, historical reviews, original research articles, case reports, letters to the editor, clinical teaching cases, neuroradiology highlights, neuropathology highlights, neuropsychiatry highlights, autobiographies, conference

\section{Dovepress}

proceedings, abstracts and book reviews. The manuscript management system is completely online and includes a very quick and fair peerreview system, which is all easy to use. Visit http://www.dovepress.com/ testimonials.php to read real quotes from published authors.

Submit your manuscript here: http://www.dovepress.com/journal-of-parkinsonism--restless-legs-syndrome-journal 